\title{
A New Binary Mathematical Programming Problem Model for Mobile
} Communication

\author{
Abdul-Rahim K. Alharithi \\ College of Administration and Economics \\ University of Sulaimany
}

Received on: 19/6/2007
Dana A. Mahmood

Sanatel Company

Accepted on: 4/11/2007

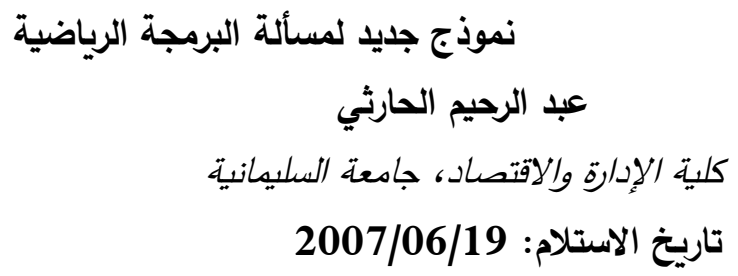

كلية الإدارة والاقتصاد، جامعة السليمانية

دانا محمود

شركة سناتل

تاريخ القبول: 2007/11/04

\section{ABSTRACT}

The aim of this study is to determine the number of base slatlons and capacity of each base station to satisfy the increased traffic demand and serving all traffic demand areas with a sufficient number of base stations, which is using a minimum number of base stations in order to minimize the total cost.

The problem is formulated as a binary linear program ming problem, and is solved by a specific algorithm. For this reason, we have built a specific algorithm to solve the model, with programming this algorithm by using Microsoft Access using Visual Basic applications, which has ability to have a decision about any changes and fluctuations.

Advantages of the algorithm can obtain best (optimum) solution with least steps.

Computational results show that the proposed model is highly effective and it is applicable, as well.

For (30) candidate base stations and (15) TDAs, we have applied the model with low costs differ by (10 unit $\$)$ with satisfying the required demand for all TDAs.

Keywords: Programming Problem, Mobile Communication.

Introduction:

In network planning [1]' 'cell planning" for mobile eommuni cation systems, we need to consider the traffic demand to cover a specific region, availability of base station sites [3]. available channel capacity at each base station and the service quality at various traffic demand areas (TDA s), selection of good base station siles among candidate locations and channels for regions that we want to submit (provide) mobile services (horizontally expansion), or increasing channels (capacity) for traffic demand areas that required (vertically expansion), all above considerations will result in 
acceptable coverage performance at base stations both In coverage area and signal quality. [4]

In this research, we combine these two expansions (vertically and horizontality) and working on them together.

\section{The problem and the aim of the study:}

The problem is how the mobile telecommunication companies can Increase the capacity of required and coverage area for the regions in which the services exist or originally has no services respectively. Because the mobile users increase day after day, serving these areas with minimum cost [6] can be achieved through using minimum number of base stations, This study is aimed at building a mathematical model [5 ] [71 and how it arises in. practice, then solving this model as well as giving interpretation of Its solution finely to provide one of the best methods to improve the services accepted by the consumer in order to expand the network vertically and horizontally with minimizing the total cost.

\section{Assumptions of the study:}

1. In this research we assume that all traffic demand areas can be serviced by the base stations with number of base stations greater than the number of traffic demand areas.

2. For those traffic demand areas that required more than one base station we introduce all types of base stations and allowable configurations for all these traffic demand areas with representing the costs of all these types.

3. For the existing base stations in service we again introduce it to the objective function, because any base station which is old or new. It costs the company and can be used in new or another existing traffic demand areas.

4. In this study we assume that channel interference is negligible For all types of interference.

5. We assume that the potential location of each candidate base station is known.

6. The researcher assumes that the relation between the cost and capacity is linear.

\section{Parameters used in file model:}

Let $(\mathrm{N})$ denote the set of traffic demand areas that we want to increase capacity and submitting services among $(\mathrm{N})$ that (originally has> no services. (Both cases for expansion arc together), for tower or site construction (base station installation). And let (B) denote the set of 
candidate base stations tor installation (contains both new and old base stations), due to cost $\left(c_{b}\right)$ and capacity $\left(m_{b}\right)$ to cover $\bullet$ traffic demand areas (N). That is:

$c_{b}$ : Cost of base station (b) belongs to (B).

$m_{b}$ : Capacity of base station (b) belongs to (B).

The traffic demand for services In traffic demand areas $(\mathrm{N})$ is denoted by $\left(d_{i}\right)$, (the number of channels required to service the mobile subscribers in each traffic demand area (i) where (i) belongs to (N)., or can be measured by Erlang number of subscriber in each traffic demand area), at an acceptable service level (GOS: grade of service) say $(\alpha)$, that is:

$\alpha$ : is the percentage of allowed congested calls due to unavailability of traffic channels.

When a subscriber in location area $(i \in N)$ is serviced by a base station $(b \in B)$, the base station must transmit with sufficient power so that the subscriber's mobile will receive it at the target power level $(p(i, b))[2]$, that is:

$p(i, b)$ : received power at base station $(b \in B)$ which transmitted from traffic demand area $(i \in N)$.

Due to attenuation, the signal transmitted weakens over the path from the mobile to the tower (base station) based on the relative location of the origin and destination, which depend on distance, topology, local conditions ...

According to the type of base stations $(b \in B)$, (QoS. quality of service) is the minimum required power level at each base station $(b \in B)$ for covering traffic demand area $(i \in N)$.

\section{Decision variables used in the model:}

The decision variables in our model include binary variables, the decision to select a base station $(b \in B)$ from candidate base station represented by variable $\left(x_{b}\right)$ which is one if selected, and zero if not.

The binary variables $\left(y_{i b}\right)$ denote serving traffic demand area $(i \in N)$ by base station $(b \in B)$. thus, $\left(y_{i b}\right)$ is one if traffic demand area $(i \in N)$ is serviced by base station $(b \in B)$, otherwise $\left(y_{i b}\right)$ is zero.

\section{Problem formulation:}

Consider that mobile users are distributed over a specific region composed of $(\mathrm{N})$ traffic demand areas (TDA $s$ ). Each traffic demand area $\left(T D A_{i}\right)$ has a demand $\left(d_{i}\right)$, for $\mathrm{i}=1,2 \ldots \mathrm{N}$. Assume that we have (B) existing base station denoted by $\left(B S_{b}\right), b=1,2 \ldots B$, like this $(\mathrm{B}>\mathrm{N})$, to satisfy 
covering and increased traffic demand, where the potential location of each candidate base station $\left(B S_{b}\right), b=1,2 \ldots \mathrm{B}$ is known.(By fifth assumption).

Let $\left(c_{b}\right)$ and $\left(m_{b}\right)$ be the cost and capacity of each candidate base station $(b \in B)$ respectively, where the cost $\left(c_{b}\right)$ is linear- to the capacity $\left(m_{b}\right)$. (By sixth assumption).

To formulate the problem, we introduce two types of binary variables: Let $\left(y_{i b}\right)$ be the variable of connection between $\left(T D A_{i}\right)$ and $\left(B S_{b}\right)$, like this:

$y_{i b}=\left\{\begin{array}{lr}\{1 & \text { if } B S_{b} \text { cover } T D A_{i} \\ 0 & \text { otherwise }\end{array}\right.$

Let $\left(X_{b}\right)$ be the selection variable of candidate $\left(B S_{b}\right), \quad b=1,2 \ldots B$

$x_{b}=\left\{\begin{array}{lr}\{1 & \text { if } B S_{b} \text { is selected } \\ 0 & \text { otherwise }\end{array}\right.$

\section{The objective function:}

The objective of the problem is to minimize the cost of base stations (Old and New).

Thus the objective function of the problem is:

Minimize $\sum_{b=1}^{B} c_{b} x_{b}$

Note:

The cost of covering $\left(T D A_{i}\right)$ by $\left(B S_{b}\right)$, assumed zero in objective function, because the important and applicable work is to minimize the base station cost. so we Focus on it. (i.e. Minimize $\sum_{b=1}^{B} c_{b} x_{b}$ )

\section{The constraints:}

1. Because of the first and second assumptions, we can make one to one correspondence between the traffic demand areas and base stations; (any $\left(T D A_{i}\right)$ can be covered by one base station which is selected).

The result is:

$\sum_{i=1}^{N} y_{i b} \leq x_{b} \quad \forall b \in B$

$\sum_{b=1}^{B} y_{i b} \quad \forall i \in N$

The above constraints (4) and (5) guarantee that all traffic demand areas are covered.

2. The coverage of existing base station may change due to the expected traffic demand and increased traffic demand from $\left(T D A_{i}\right), i \in N$, and cells type. So it is important to satisfy the 
coverage limit of the total traffic demand areas (expecting and increasing). For this we have a minimum portion which is to be covered by selected base stations, namely: The minimum portion covers the minimum required trafic demand in acceptable level due to an acceptable grade of service, (GOS). Mathematically: there is at least $\alpha$, where $(0 \leq \alpha<1)$ of the total traffic demand has to be covered by selected base stations in the traffic demand areas. Thus:

$\sum_{b=1}^{B} \sum_{i=1}^{N} m_{b} y_{i b} \geq(1-\alpha) \sum_{i=1}^{N} d_{i}$

3. Since all traffic demand areas $\left(T D A_{i}, i \in N\right)$. should be covered by a base station, the traffic demands supported by the base stations cannot exceed the capacity of the selected one. i.e.

$\sum_{i=1}^{N} d_{i} y_{i b} \leq m_{b} x_{b} \quad \forall b \in B$

4. The last two sets of constraints provide the domains for the variables:

$x_{b} \in\{0,1\} \forall b \in B$

$y_{i b} \in\{0,1\} \quad \forall i \in N \& \forall b \in B$

\section{Application of the model:}

For testing the validity of the model for expanding a mobile network problem which is indicated above, for any specific problem we need to determine the following input data:

1. The number of traffic demand areas $(\mathrm{N})$. the existed and the new areas.

2. The required traffic demand $\left(d_{i}\right)$, for each traffic demand area $(i \in N)$ due to GoS value $(\alpha)$ which is determined by the mobile operator.

3. The number of base stations (B), due to allowable configurations with capacity $\left(m_{b}\right)$ and cost $\left(c_{b}\right)$ for each one. i.e. for each $(b \in B)$.

\section{Solving the model for a specific problem:}

In the problem, we take the case of covering a number of traffic demand areas with existing base stations due to required traffic demand where the input parameters are described below:

1. Number of $T D A_{i}, N=15$ (for both existed and new sites together), and we name each $T D A_{i}$ as $\left(A_{1}\right.$, for $T D A_{1}, A_{2}$, for $T D A_{2}, \ldots A_{N}$, for $\left.T D A_{N}\right)$ that is $(i=1,2, \ldots \ldots \ldots, 15)$ in our case.

2. The traffic demand $\left(d_{i}\right)$ for each $(i \in N)$ can be obtained according to GoS value $(\alpha)$ where $(\alpha=0.02)$ in our problem. 


\section{Available and required traffic demand}

\begin{tabular}{|c|c|c|}
\hline Traffic demand area & Availabe TCH & Require TCH $\left(\right.$ demand $\left.d_{i}\right)$ \\
\hline$A_{1}$ & 90 & 122 \\
\hline$A_{2}$ & 90 & 45 \\
\hline$A_{3}$ & 90 & 164 \\
\hline$A_{4}$ & 90 & 103 \\
\hline$A_{5}$ & 90 & 47 \\
\hline$A_{6}$ & 90 & 46 \\
\hline$A_{7}$ & 90 & 126 \\
\hline$A_{8}$ & 42 & 42 \\
\hline$A_{9}$ & 42 & 63 \\
\hline$A_{10}$ & 90 & 59 \\
\hline$A_{11}$ & 90 & 51 \\
\hline$A_{12}$ & 90 & 46 \\
\hline
\end{tabular}

Analysis of traffic demand for new sites: $\left(A_{13}-A_{15}\right)$

To determine the required traffic. The number of subscribers (expected) and the (GoS) value have to be known.

As the quality of service is constrained, each area is determined (supported by three cells or sectors in standard case). This means that we do not introduce the number of cells, only focus on the number of subscribers and Erlang per subscriber (The Erlang $E$ is a unit of measurement of traffic intensity) that can be supported by the base station with a given existing number of $\mathrm{TCH}$.

Erlang (E) can be calculated with the following formula :

$$
A=\frac{n \times T}{3600} \text { Erlang }
$$

Where:

$A=$ Offered traffic from one or more users in the system.

$n=$ Number of calls per hour.

$T=$ Average call time in seconds.

Due to Erlang value in our problem we have a standard relation between supporting the maximum number of subscribers by a base station and a specific number of TCH, as Follows: A base station with $90 \mathrm{TCH}$ can support (2500) subscribers for our Erlang so from this proportion and expected number of subscribers we can compute the required demand (approximately) for the new areas $\left(A_{13}-A_{15}\right)$ 
For $A_{13}$

\section{Number of Subscribers}

2500

1000

\section{Required TCH}

90

$d_{13}$

$d_{13}=\frac{90 \times 100}{2500}=36$, which is the required TCH for $A_{13}$

By the same way for $A_{14}$ and $A_{15}$ we have:

$d_{14}=72$ (2000 subscriber)

$d_{15}=63$ (1750 subscriber)

3. The number of base stations in our problem with allowable configurations are (30) base stations, that is $(B=30)$. So $(b=1,2, \ldots \ldots \ldots, 30)$ The following table contains cost and capacity for each base station:

\section{Cost and capacity' for candidate base stations}

\begin{tabular}{|c|c|c|}
\hline$B_{b}$ & $m_{b}$ & $c_{b}$ \\
\hline 1 & 42 & 20 \\
\hline 2 & 42 & 20 \\
\hline 3 & 42 & 20 \\
\hline 4 & 42 & 20 \\
\hline 5 & 60 & 25 \\
\hline 6 & 60 & 25 \\
\hline 7 & 60 & 25 \\
\hline 8 & 60 & 25 \\
\hline 9 & 60 & 25 \\
\hline 10 & 90 & 40 \\
\hline 11 & 90 & 40 \\
\hline 12 & 90 & 40 \\
\hline 13 & 90 & 40 \\
\hline 14 & 90 & 40 \\
\hline 15 & 115 & 45 \\
\hline 16 & 115 & 45 \\
\hline 17 & 138 & 60 \\
\hline 18 & 138 & 60 \\
\hline 19 & 138 & 60 \\
\hline 20 & 160 & 65 \\
\hline 21 & 160 & 65 \\
\hline 22 & 160 & 65 \\
\hline 23 & 186 & 80 \\
\hline 24 & 186 & 80 \\
\hline
\end{tabular}




\begin{tabular}{|c|c|c|}
\hline 25 & 200 & 85 \\
\hline 26 & 200 & 85 \\
\hline 27 & 240 & 100 \\
\hline 28 & 270 & 105 \\
\hline 29 & 282 & 120 \\
\hline 30 & 320 & 125 \\
\hline
\end{tabular}

\section{Our Algorithm:}

We use a specific algorithm which is built for solving the problem by using Microsoft Access for inputting the parameters and using visual basic applications tor programming the algorithm.

The mechanism of our algorithm and how it is applied is described below:

Step 1: Arrange candidate base stations in non decreasing order due to the capacity.

We may have more than one base stations which are of the same type and capacity.

Step2: For $b=\hat{b}$, if $m_{b} \geq d_{i}$ for some $\mathrm{i}$, set $x_{b}=1$ go to step4.

From this step we see that if there exist a base station which covers at least one TD for a given TDA, set it equal to 1 .

Step3: If $m_{b}<d_{i}$ set $b=\hat{b}+1$ and Go to step2.

This step indicates that if a base station does not cover any TDA. then remove it from the list. that is set it equal to zero. and go to check another base station in Slep2.

Step4: Choose $\left\{\operatorname{Max}\left(m_{b}-d_{i}\right)\right\}$ for some $i$ like $i$ and set $y_{i \hat{b}}=1$.

This means, the selected base station in Step2 should cover a TDA which have the minimum TD among the areas, and set it equal to 1 .

Step5: Set $y_{i \hat{b}}=0$ for $b \neq \hat{b}$ and set $y_{i \hat{b}}=0$ for $i \neq i$.

For this TDA that is covered by a base station in Step4, no other base station should be chosen for this TDA and vice versa, that is set all other variables equal to zero.

Step6: If all demand areas arc covered, go to step7 and stop, otherwise, set $b=\hat{b}+1$, go to step2. This means that if all areas arc covered, then calculate the total cost for all the selected base stations and stop, otherwise continue to cover the remaining areas.

Step7: Calculate $Z=\sum_{i=1}^{B} c_{b} x_{b}$ for all $x_{b}=1$, the total cost of all the selected base stations. 


\section{Flow Chart}

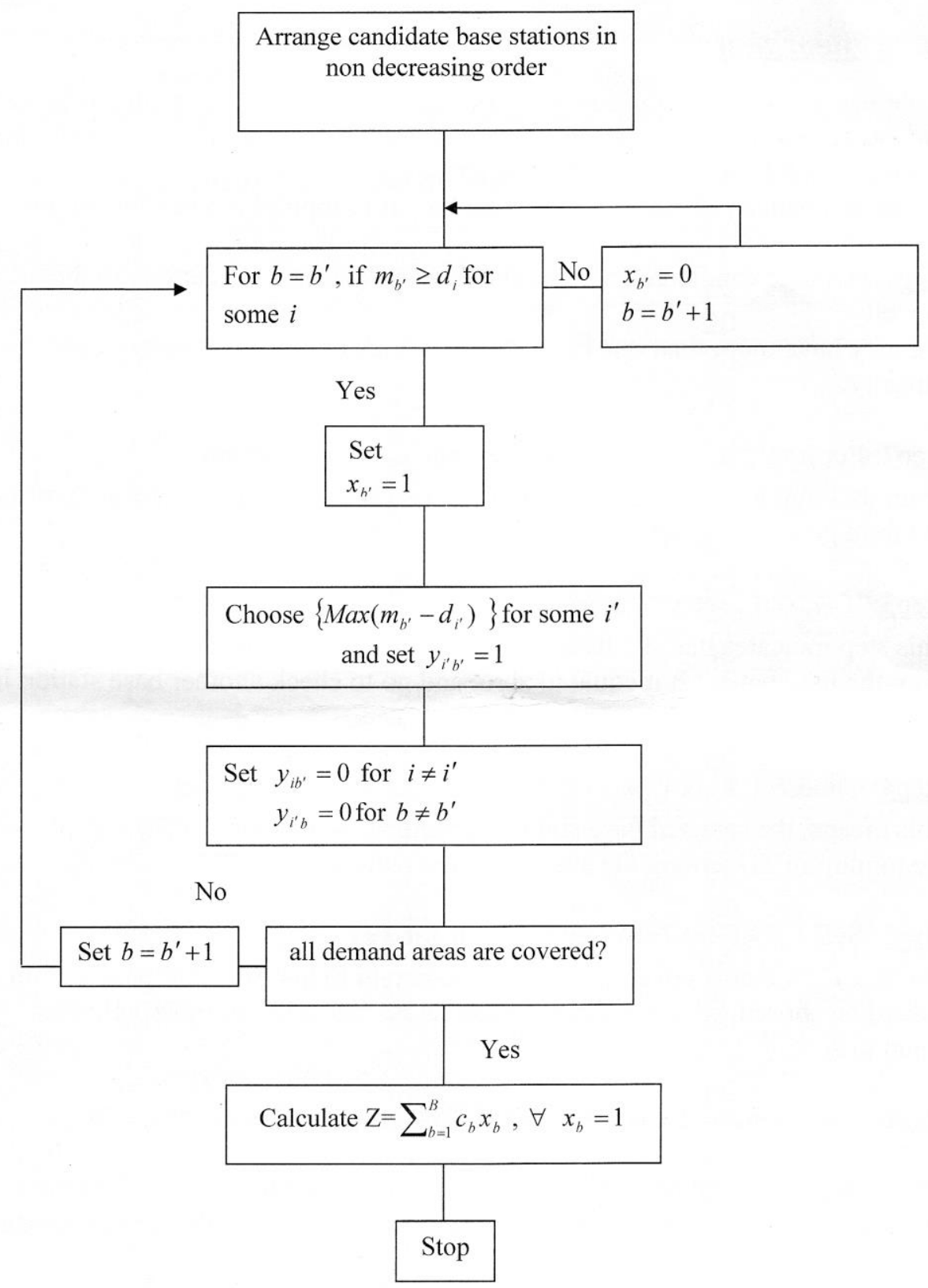

Figure (1): Flow Chart of the new algorithm 


\section{Computational results:}

After solving the problem, we have got the following results:

1. If we compare the total cost. we see that the required cost is lesser than the available cost by 10 units (\$), while we apply the problem tor (15) TDAs.

2. Some TDAs require more capacity than the available capacity. Like ( $\left.A_{1}, A_{3}, A_{4}, A_{7}, A_{9}\right)$, which is important, to satisfy the required demand.. and then preserving the subscribers registration.

3. We see that there exists TDAs which can be covered by available base station., which remains as above situation, like $\left(A_{8}\right)$.

4. Some TDAs require less capacity than the available capacity. like ( $\left.A_{2}, A_{5}, A_{6}, A_{10}, A_{11}, A_{12}, A_{13}, A_{14}, A_{15}\right)$, that is we can cover each one in acceptable (GOS) value with less costs, which is very important. if it is applied to a big network.

5. About our algorithm, we see that this algorithm can give an exact solution if compared to the other optimization solvers like WinQSB.

6. Our program has an ability to compute any fluctuations or any changes in the required demands, that is sensitivity analysis, which increases other base station or increases TDA, or changes costs for the base stations and vice versa.

\section{Conclusions:}

From our study, after solving the problem, we have come to the following conclusions:

1. From this study we observe that preparing an optimization team in telecom companies is one of the major works that affect the strategy of the plans to get better decision for problems.

2. For covering some areas and to preserve the cost reduction much more, the companies can provide specific type of base stations for locations that the ratio of increasing subscribers is bounded like rural regions.

3. Our model while be applied to vertical and horizontal expansion together, it is applied to each one of them separately as well. 


\section{REFERENCES:}

[1] Chae Y.Lee.Member IEEE \& Hyon G.Kang."'Cell planning with capacity expansion in mobile communications, A Tabu search approach". IEEE Transactions on vehicular technology. Vol.49. No.5. Sep.2000.

[2] I. Harris. Data In the GSM cellular network. In D. M. Balston and R.C.V. Macario,editors, Celluar Radio System. Artech House, Boston, Copyright 0 John Scourias 1993.

[3] M. Bezicr el al. GSM base station system. Electrical Communication, John Scourias. $2^{\text {nd }}$ Quarter 1993.

[4] Michel Mouly and Marie-Bernadette Pautet. the GSM System for Mobile Communications. Published by the authors. John Scourias, 1992.

[5] Prem Kumar Gupta,"Operations Research An Introduction", 2nd hd. Chand \& Company LTD, Ram Nagar, New Delhi-1 10055. 2002.

[6] Taha.H.A.," Operations research an Introduction", $5^{\text {th }}$ Ed. Macmillan publishing Co. Inc, Singapore, 1992.

[7] Wayne L. Winston, "Operations Research Applications and Algorithms", $4^{\text {th }}$ Ed. Thomson Learning Academic Resource Center. Inc. 2004 